\title{
Microneedling dilates the follicular infun
and increases transfollicular absorption of liposomal sepia melanin
}

This article was published in the following Dove Press journal:

Clinical, Cosmetic and Investigational Dermatology

26 June 2015

Number of times this article has been viewed

\author{
Gabriel Serrano' \\ Patricia Almudéver ${ }^{2}$ \\ Juan M Serrano 3 \\ Julio Cortijo ${ }^{2}$ \\ Carmen Faus' \\ Magda Reyes' \\ Inmaculada Expósito ${ }^{3}$ \\ Ana Torrens ${ }^{3}$ \\ Fernando Millán' \\ 'Clínica Dermatológica Serrano, \\ ${ }^{2}$ Department of Pharmacology, \\ University of Valencia, ${ }^{3}$ Research and \\ Development Department, Sesderma \\ Laboratories, Valencia, Spain
}

\begin{abstract}
Encapsulation of chemicals in liposomes and microneedling are currently used techniques to enhance the penetration of several substances through skin and hair. In this study, we apply a liposomal melanin-fluorescein compound to an ex vivo model of human skin, using a new electrical microneedling device (Nanopore turbo roller). The product was applied by hand massage (A) or with the assistance of the electrical roller for 2 minutes (B). An additional test was performed free of product and with only the E-roller (C). Histological changes and product absorption were evaluated by optical and fluorescent microscopy 60 and 90 minutes after the treatment. Site B showed larger deposits of melanin-fluorescein at superficial and deep levels of hair structures in comparison to site A. Light, epidermal deposits of the melanin-fluorescein complex were also observed. Sites B and C showed a significant widening (47\%) of the follicular infundibulum which could explain the increased penetration of the formulation. Microneedling also removed the scales and sebum residues in the neighborhood of the infundibulum. Targeting hair follicles with melanin may be useful to dye poorly pigmented hairs, improving laser hair removal. The procedure accelerates the delivery of melanin into hair structures allowing an even absorption, larger pigment deposits, and deeper penetration of the formulation into the hair.
\end{abstract}

Keywords: liposomes, melanin, microneedling, follicular infundibulum, hair removal

\section{Introduction}

To improve the efficiency of topical penetration of substances is important for the development and optimization of medicines and cosmetics. The skin penetration occurs through three possible routes: transcellular, intercellular, or follicular. ${ }^{1,2}$ The skin represents an important barrier to the penetration of exogenous substances into the body and also a potential route for the transport of functional active principles into the skin and hair. In the past, it was assumed that the intercellular route was the only relevant penetration pathway for topically applied substances. The transfollicular path had been largely ignored because hair follicles constitute only $0.1 \%$ of the total skin. Recent results on follicular penetration emphasize that the hair follicles represent a highly relevant and efficient penetration pathway and reservoir for topically applied substances. ${ }^{1}$ Hair follicles are an interesting target site for topical applications. They are closely surrounded by capillaries and antigen-presenting cells and are associated with the sebaceous glands and host the stem cells in the bulge region. In the face or scalp, there are between 500 and 1,000 pilosebaceous units per $\mathrm{cm}^{2}$, with follicular openings of 50-200 $\mu \mathrm{m}$ of diameter, representing $10 \%$ of the total area, while the rest of skin represents only $0.1 \%{ }^{3}$ This pathway is well accepted for the treatment of diseases such as androgenic alopecia, acne, increased tumor cell proliferation, and hair growth control.
Correspondence: Gabriel Serrano

Clínica Dermatológica Serrano,

Grabador Esteve 24,

46004 Valencia, Spain

Tel +346I58I0I59

Email gabriels@sesderma.com 
Melanin occurs naturally in the skin and gives skin and hair their color. Laser hair removal devices rely on selective photothermolysis using melanin as a chromophore., ${ }^{4,5}$ Loading melanin into the hair follicles could improve the effectiveness of the laser in subjects with blonde, gray, or white hairs. However melanin penetration through the skin and hair is hindered due to its high molecular weight and its hydrophilic nature.

Melanin encapsulation in liposomes may improve its penetration inside hair structures. ${ }^{6,7}$ Recently, skin needling has been described as a new technique also able to increase transdermal drug absorption. ${ }^{8,9}$ In this study, a new electrical microneedling device was used to apply sepia melanin liposomes to human hairy skin in order to improve the transfollicular absorption. ${ }^{10}$

\section{Objective}

To study the transfollicular penetration of sepia melanin encapsulated in nanoliposomes (Melaser solution, Sesderma, Valencia, Spain) applied with a new microneedling electrical device on an ex vivo model of human skin.

\section{Materials and methods}

\section{Products}

A $5 \mathrm{~mL}$ suspension of sepia melanin encapsulated in positively charged phosphatidylcholine liposomes (Melaser solution, Sesderma) was mixed with an equal amount of fluorescein sodium salt (Sigma-Aldrich, St Louis, MO, USA)-loaded liposomes at a concentration of $1 \mathrm{mg} / \mathrm{mL}$ in a clean aluminum container. The product was stirred for 5 minutes keeping it well protected from the light, until a homogenous mixture was obtained. The liposome preparation presented a unimodal size distribution with a diameter between 80 and $120 \mathrm{~nm}$, a polidispersity index below 0.12 , and a zeta potential of $47.79 \mathrm{mV}$. The size of the unillamelar nanoliposomes was between 80 and 120 $\mathrm{nm}$ in diameter (Delsa Nano C, particle analyzer, Beckman Coulter Inc., Pasadena, CA, USA). The melanin concentration was $0.52 \%$, and the $\mathrm{pH}$ of the solution was 8.5 .

\section{Electrical roller}

The Nanopore turbo roller was from Mediderma, Valencia, Spain. This device has a console, an L-shaped hand-piece with an integrated high performance electrical motor, and a microneedle module which reaches between 50 and 150 pulses per second through electrical pulses (300-900 per second). The needle module ( $5 \mathrm{~mm}$ wide) has 6 micro needles, each $1.5 \mathrm{~mm}$ apart which may be adjusted from
0.25 to $1.5 \mathrm{~mm}$ (maximum length). Needle thickness at maximum penetration point is $0.15 \mathrm{~mm}$, and the needle tip radius is $2 \mu \mathrm{m}$. For this study, length of the microneedles was set at $0.5 \mathrm{~mm}$ and pulses to 150 pricking channels $/ \mathrm{min} /$ skin spot. Application was performed through continuous circular movements (Figure 1).

\section{Fluorescent microscope}

A Leica DM6000B microscope (Wetzlar, Germany), equipped with a digital camera DFC480 and a Leica GFP filter was used for the fluorescence assessment. Excitation of the sample was carried out with $470+40 \mathrm{~nm}$ blue light. The fluorescent emission obtained was in the visible green waveband at 525+50 $\mathrm{nm}$. Assessment of the fluorescence intensity at the two time periods was determined by comparing images. Evaluation was done by scoring specimens from 0 to 8 (adapted from the Ashcroft scale for pulmonary fibrosis) to determine the amount of product present in the specimens corresponding to each of the time frames. A score of 0 indicates no staining of the follicular duct (nonexposed hair follicle). A value of 8 means a totally pigmented hair follicle.

\section{Procedure}

The clinical study protocol was prepared, reviewed, and approved by the General Hospital of Valencia Ethics Committee. Surplus healthy Caucasian skin containing hairs were selected for the study, from patients between 18

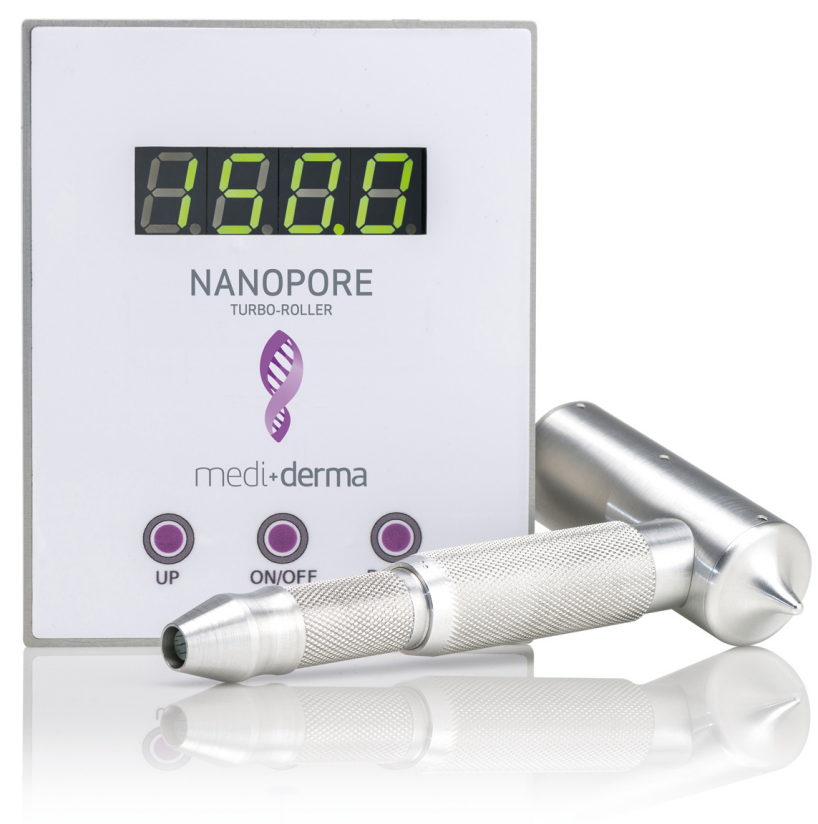

Figure I Nanopore turbo roller. 
and 60 years of age who had been operated in the plastic surgery department at the Valencia General Hospital and who voluntarily accepted their inclusion in the study. Sepia melanin was applied over two $5 \mathrm{~cm}^{2}$-skin sites with the hand (A) and with an electrical roller as described below (B). Another skin site of the same area was treated with the electrical device, but with no melanin (C). After application, a 60- and 90-minute contact period was left based on previous experiences. After these incubation times, the preparation was carefully removed from the surface of the skin with $0.9 \% \mathrm{NaCl}$-solution-moistened gauzes. Finally, biopsy punches were done around the hair sites exposed to the melanin solution. Samples were included in the embedding medium for cryostat. The samples obtained were kept in the dark for their fluorescent evaluation.

The experimental conditions were as follows:

A) Manual application of $1 \mathrm{~mL}$ melanin liposomes for

2 minutes followed by a single hand massage for other additional 2 minutes.

B) Application of $1 \mathrm{~mL}$ melanin liposomes with e-roller for 3 minutes, followed by manual massage for 2 minutes.

C) Application of only e-roller for 3 minutes followed by hand massage for 2 minutes.

Skin samples were taken after 60 and 90 minutes. Histological changes evaluated in the study include:

1) Structural changes induced by microneedling with hematoxylin-eosin staining.

2) Quantification of the diameter of the hair infundibulum which corresponds, in a histological section, to the length of the opening of the hair infundibulum, through software for image analysis (Imagej ${ }^{\circledR}$, National Institutes of Health, Bethesda, MD, USA).

3) Quantification of melanin and fluorescein in the deepest hair follicles (hair follicles without granular layer/ sebaceous glands) by Fontana-Masson staining ${ }^{11}$ and by fluorescent microscopy respectively.

Assessment of the fluorescence intensity at the two time periods was determined by comparing images. Three independent evaluators scored the degree of fluorescence on the fluorescent microscopy images using the adapted Ashcroft scoring method (adapted from the Ashcroft scale for pulmonary fibrosis). Evaluation was done by scoring specimens from 0 to 8 to determine the amount of product present in the specimens, corresponding to each of the time frames. A score of 0 indicates no staining of the follicular duct (nonexposed hair follicle). A value of 8 means a totally pigmented hair follicle.

\section{Results}

Structurally, the skin treated with the e-roller showed a wider infundibulum size $(275.5 \pm 53.1 \mu \mathrm{m}, \mathrm{n}=7)$ than untreated skin $(128.3 \pm 13.1 \mu \mathrm{m}, \mathrm{n}=7)$ (Figures 2 and 3 ). This occurred without damage to the epidermis and without affecting other skin structures. Epidermal melanin absorption was observed after 60 and 90 minutes of skin contact to the product and was manifested by moderate deposits of melanin in the epidermal layer (Figure 4). Isolated, light deposits of melanin were also observed in the skin treated with manual massage. Fontana-Masson stains and fluorescent microscopy studies showed presence of significant deposits of melanin and fluorescein in the deeper hair follicles and hair shafts only with the electrical roller device (Figure 5). The intensity of the fluorescence and melanin staining was slightly higher in the samples taken 90 minutes after the application of the compound compared with those taken 60 minutes after (score 8 vs 6). Hand application of Melaser showed very light melanin deposits inside hair follicles at 60 (score 2) and 90 minutes (score 3). No melanin deposits were observed in the sites treated only with the e-roller.

\section{Discussion}

In this paper, we present the results of combining two techniques to improve the penetration of a sepia melaninfluorescein compound across skin and hair. Melanin is the natural chromophore inducing photothermolysis in laser hair removal. It has a high molecular weight and a hydrophilic nature making it very difficult to permeate through skin and hair. Liposome formulations are known to improve mainly transappendageal penetration. ${ }^{12}$ The encapsulation of melanin in nanoliposomes increases its solubility in lipids, facilitating

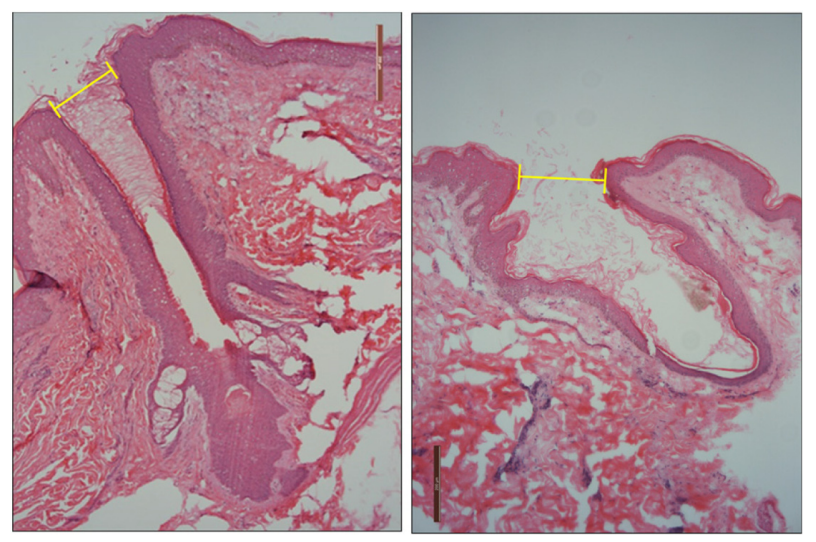

Figure 2 Pictures of hair follicles found in untreated skin (left) and e-roller treated skin (right). (Hematoxylin-eosin stain, at I0×.) 


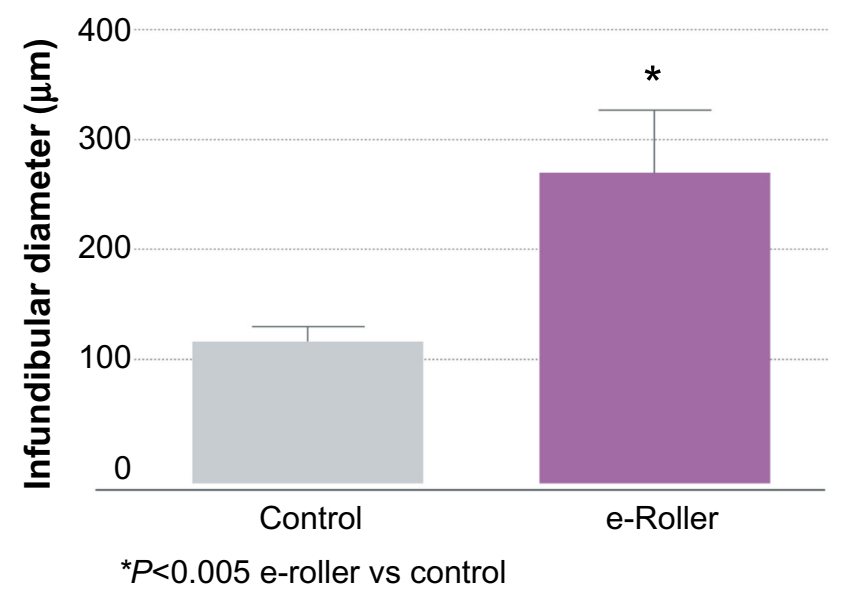

Figure 3 Graphical representation of the average diameter of the infundibulum of the massaged skin (gray bar) and the e-roller treated skin (purple bar). Note: Number of samples $=10$.

its absorption. In the present study, application of melanin by the e-roller had a better effect in carrying the pigment inside deep hair structures in comparison to application by hand massage. Only very small isolated deposits of this compound were seen in superficial hair follicles in the sites where melanin was applied by hand massage (score 2 vs 3 ). On the contrary, the application of melanin with the e-roller showed significant deposits of melanin and fluorescein in the deeper hair follicles and hair shafts (Figure 5) both at 60 and 90 minutes (score 6 vs 8). Microneedling is a physical technique that induces mechanical alterations of the horny barrier and creates channels or pores of micron-sized dimensions in the skin, enabling the delivery of a broad range of molecules, including proteins, which would otherwise not pass through the intact skin. ${ }^{13-15}$ These microchannels spontaneously close after 10 minutes, and the epidermal barrier remains intact, ${ }^{9}$ which explain why postoperative infections do not occur after the procedure. This is a great advantage when this therapy is compared with other treatments such as fractional lasers that induce alterations in the epidermis.
Previous studies have shown that microneedling increases the skin absorption of different compounds. ${ }^{16-19}$ In a clinical study from India, the efficacy of tranexamic acid microinjections was compared with tranexamic acid applied by microneedling in patients with melasma. ${ }^{16}$ The patients treated with microneedling showed better improvement that those treated by microinjections. The authors concluded that microneedling delivered the medication more evenly and deeper into the skin. In another clinical study of two patients with alopecia areata, the application of triamcinolone acetonide by microneedling facilitated the absorption of the drug with its known immunomodulatory effect. ${ }^{17}$ One more advantage of this combination in this condition could be that the collagen induced by microneedling may counter the possible atrophy caused by triamcinolone. Microneedling has also been used to enhance the penetration of minoxidil and to stimulate hair stem cells and growth factors in instances of alopecia androgenetica. ${ }^{18}$

Microneedling may be also useful to enhance the penetration of ALA (amino levulinic acid) or MAL (methyl amino levulinate) in photodynamic therapy (PDT). ${ }^{19,20}$ Clementoni et $\mathrm{al}^{19}$ treated 21 patients with photoaging by using PDT with $630 \mathrm{~nm}$ light and broadband-pulsed light after multiple passes with a manual roller and 1-hour ALA incubation period. ${ }^{19}$ This "pretreatment" prior to application of ALA appeared to be well tolerated and allowed for even absorption and, perhaps, deeper penetration of ALA following the defined incubation period. Torezan et al ${ }^{20}$ also used microneedling-assisted PDT to enhance the penetration of MAL into the epidermis in patients with solar keratosis. Improvement was more pronounced on the microneedling-assisted side. The authors concluded that this method can produce cosmetic results superior to those of conventional MAL-PDT for improving photodamaged skin, with similar results for actinic keratosis reduction.

The type of device, the needle assembly, the number of microneedles and its length, the number of hits per second,
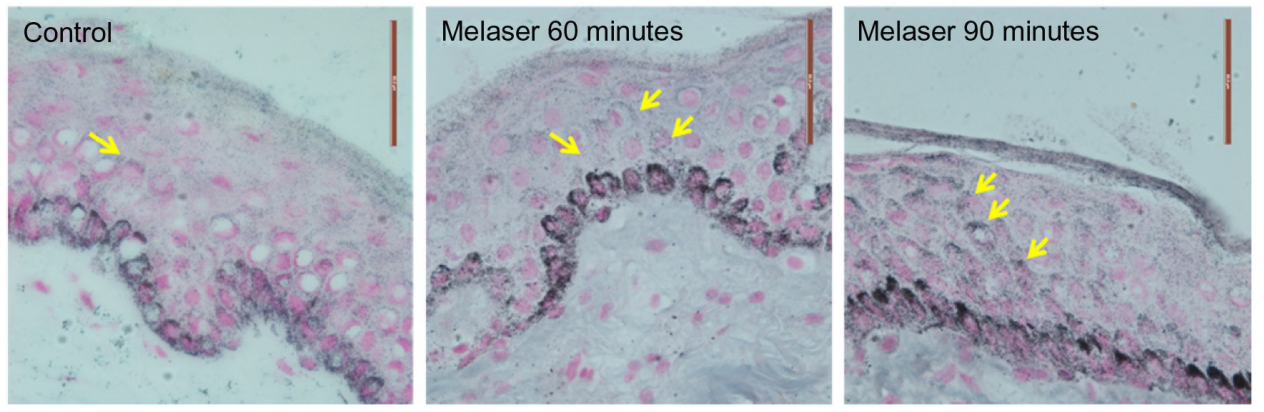

Figure 4 Images of control (untreated) and human epidermis treated with e-roller + sepia melanin after an incubation time of 60 and 90 minutes (63×). The yellow arrows indicate light $(60 \mathrm{~min})$ and moderate $(90 \mathrm{~min})$ deposits of melanin within the lower epidermis (e-roller treated skin). 


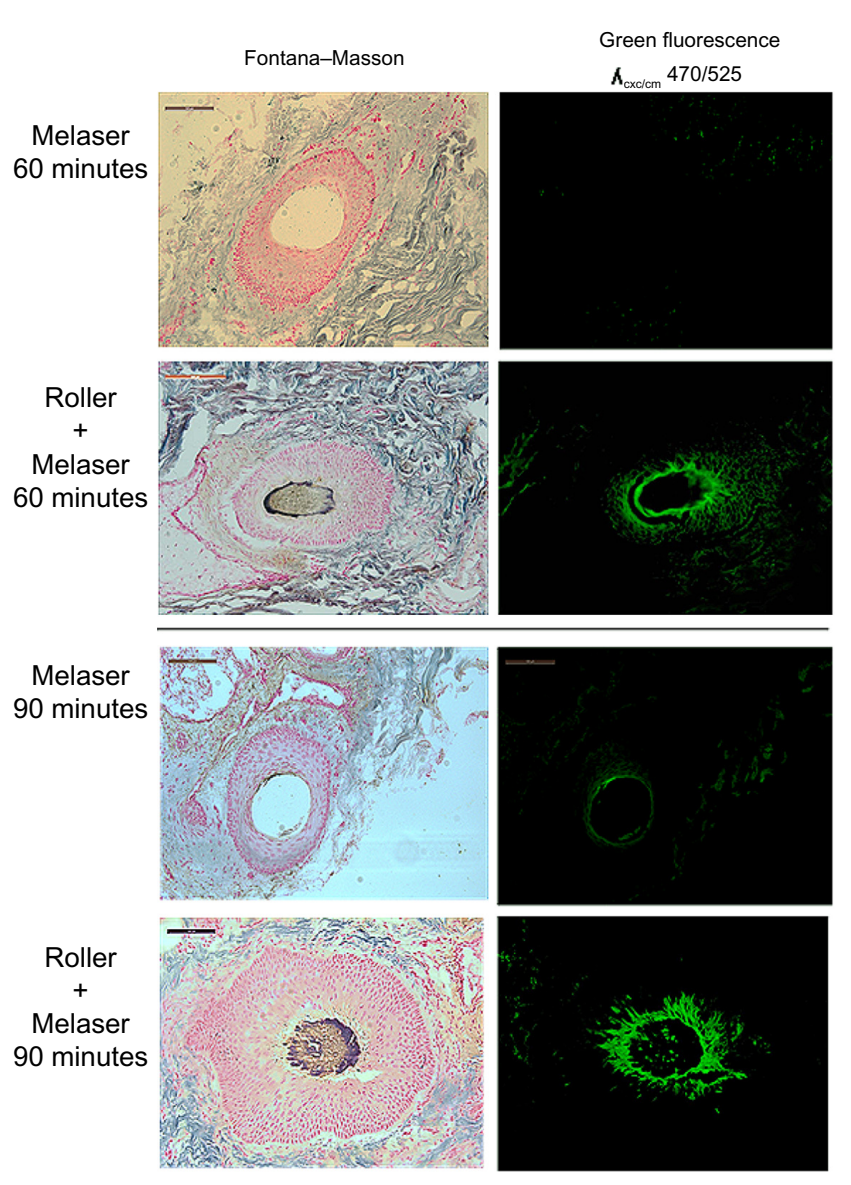

Figure 5 Presence of large deposits of melanin and fluorescein in the deeper hair follicles in the e-roller treated sites at 60 (score 6) and 90 minutes (score 8).

Notes: Hand application of Melaser showed very light melanin deposits inside hair follicles at 60 (score 2) and 90 minutes (score 3). 10x.

Abbreviations: exc, excitation; em, emission.

and the velocity severely influence the penetration depth and pore size. ${ }^{15,20-23}$ It is postulated that the electrical device notably increases the number of pores produced in the skin in a short period of time (seconds), accelerating the penetration of the product. The device can be set to a speed of 150 pulses per second, and, considering that the needle module contains 6 microneedles, a total of 900 micro channels can be created in the skin per second. This explains why increased amounts of sepia melanin entrapped in liposomes were easily incorporated inside hair structures by application of the solution with the electrical roller device. The application of the device on the skin without any product induced a $47 \%$ increase in dilation or widening of the hair infundibulum, and, when product was applied, the roller enhanced the movement of the compound inside deeper hair structures. This diffusion starts after microneedling and notably increases after 60 and 90 minutes. Needles with $0.5 \mathrm{~mm}$ length were very effective for the intended purpose. Another reason to explain the increased melanin penetration is a change in the environment of the follicular infundibulum which often contains scales and sebum residues coming in a continuous stream from the outlet of the sebaceous gland toward the follicular orifice. At this level, liposomes may stick to the scales of the hair shaft slowing down the penetration process. Microneedling could remove all this debris, acting as a brush to achieve a more effective penetration of the liposomal product.

The epidermis remains intact and allows the incorporation of a small amount of melanin 1 hour after exposure. This coincides with the results achieved in our laboratory in which absorption of $1 \mu \mathrm{g} / \mathrm{cm}^{2}$ was observed after 1 hour of exposure to liposomal fluorescein. This absorption should be further assessed in the clinical trials of the product, as this may cause injuries, such as burns, after laser exposure. Preliminary results of current studies however indicate that the procedure is safe. This may be related to the small epidermal melanin deposits and the longer wavelengths required by hair-removal lasers. Melanin has a strong absorption spectrum at $335 \mathrm{~nm}$ in the ultraviolet A range (not present in these lasers) as well as in the yellow, green, and near infrared light which have the capacity to penetrate deeper into the skin and be absorbed by hair structures. The orifices of the pilosebaceous units make up approximately $10 \%$ in areas where their density is high (face and scalp) and only $0.1 \%$ in areas where their density is low. These facts must be taken into consideration for developing topical treatments for rich, hairy areas. Targeting the hair follicles with melanin to color hair has been previously demonstrated. ${ }^{6,7}$ Liposomal melanin lotions are being employed for laser hair removal in the past years with conflicting results. In a clinical study in 16 patients with blond, gray, or white hair, a melanin liposomal spray was applied on the treatment sites 12 times a day (6 times in the morning and 6 times in the evening) for a period of 8 weeks before each laser treatment. ${ }^{24}$ Patients were advised to shave the areas 2 days before treatment and stop applying the liposomal melanin the night before laser treatment. Treatment was performed with an 800-nm diode laser with a fluence range of $22-36 \mathrm{~J} / \mathrm{cm}^{2}$ and pulse duration of $30 \mathrm{~ms}$. Mean regrowth was $83 \%$, while baseline pretreatment showed $14 \%$ reduction. The clinical outcome was disappointing because of the poor hair reduction and the high costs of the procedure. In another study, ${ }^{25} 40$ subjects with white, gray, or blonde hairs were also instructed to apply a melanin liposomal spray 6-8 times a day, 14 days prior to the treatment. Six months after the last treatment, most patients (90\%) had considerable hair reduction $(75 \%-100 \%)$. Sixty-two percent of patients achieved a permanent hair reduction (between 95\% and 100\%). In both cases, the application had several inconveniences. Application 
of melanin required large amounts of the product, it had to be applied several times a day for several weeks, and there was a lack of control in home treatments and no patient compliance. Some patients showing good results at the beginning of the treatment finally reduced the application of the lotion, which led to negative results.

The combination of Nanopore turbo roller with a melanin liposomal solution will increase the effectiveness of laser in people with blond, gray, or white hairs. The procedure will significantly reduce the application time of the melanin solution from weeks to minutes, as well as increase the amount of product deposited in the deeper hair structures, reducing the cost of the hair-removal procedure.

\section{Disclosure}

Dr Gabriel Serrano is the medical director of Sesderma Laboratories, Valencia, Spain. Juan M Serrano, Ana Torrens, and Inmaculada Expósito belong to the research and development department of Sesderma Laboratories. The authors report no other conflicts of interest in this work.

\section{References}

1. Lademann J, Knorr F, Richter H, et al. Hair follicles - an efficient storage and penetration pathway for topically applied substances. Summary of recent results obtained at the Center of Experimental and Applied Cutaneous Physiology, Charite-Universitatsmedizin Berlin, Germany. Skin Pharmacol Physiol. 2008;21(3):150-155.

2. Lademann J, Otberg N, Richter H, et al. Follikuläre Penetration. Ein wichtiger weg für topisch applizierte substanzen. [Follicular penetration. An important pathway for topically applied substances]. Hautarzt. 2003;54(4):321-323. German.

3. Meidan VM, Bonner MC, Michniak BB, et al. Transfollicular drug delivery - is it a reality? Int J Pharm. 2005;306(1-2):1-14.

4. Nanni CA, Alster TS. A practical review of laser-assisted hair removal using the Q-switched Nd:YAG, long-pulsed ruby, and long-pulsed alexandrite lasers. Dermatol Surg. 1998;24:1399-1405.

5. Liew SH, Ladhani K, Grobbelaar AO, et al. Ruby laser-assisted hair removal success in relation to anatomic factors and melanin content of hair follicles. Plast Reconstr Surg. 1999;103(6):1736-1743.

6. Hoffman RM. Topical liposome delivery of molecules to hair follicles in mice. J Dermatol Sci. 1997;14(2):101-108.

7. Hoffman RM. Topical liposome targeting of dyes, melanins, genes, and proteins selectively to hair follicles. J Drug Target. 1998;5:67-74.

8. Henry S, McAllister DV, Allen MG, Prausnitz MR. Microfabricated microneedles: a novel approach to transdermal drug delivery. J Pharm Sci. 1998;87(8):922-925.
9. Verma DD, Fahr A. Investigation on the efficacy of a new device for substance deposition into deeper layers of the skin: Dermaroller ${ }^{\circledR}$. Philipps-Universität Marburg. Institut für Pharmazeutische Technologie und Biopharmazie, 2001.

10. Liu KC, Green CR, Alany RG, Rupenthal ID. Synergistic effect of chemical penetration enhancer and iontophoresis on transappendageal transport of oligodeoxynucleotides. Int J Pharm. 2013;441(1-2): 687-692.

11. Miot HA, Brianezi G, Tamega Ade A, Miot LD. Techniques of digital image analysis for histological quantification of melanin. An Bras Dermatol. 2012;87(4):608-611.

12. Korting HC, Stolz W, Schmid Mil, Maicrhofcr G. interaction of liposomes with human epidermis reconstructed in vitro. $\mathrm{Br} J$ Dermatol. 1995:132:571-579.

13. Kaushik S, Hord AH, Denson DD, et al. Lack of pain associated with microfabricated microneedles. Anesth Analg. 2001;92:502-504.

14. Prausnitz MR, Mikszta JA, Cormier M, Andrianov AK. Microneedlebased vaccines. Curr Top Microbiol Immunol. 2009;333:369-393.

15. Prausnitz MR. Microneedles for transdermal drug delivery. Adv Drug Deliv Rev. 2004;56:581-587.

16. Budamakuntla L, Loganathan E, Suresh DH, et al. A randomised, open-label, comparative study of tranexamic acid microinjections and tranexamic acid with microneedling in patients with melasma. J Cutan Aesthet Surg. 2013;6(3):139-143.

17. Chandrashekar BS, Yepuri V, Mysore V. Alopecia areata - successful outcome with microneedling and triamcinolone acetonide. J Cutan Aesthet Surg. 2014;7(1):63-64.

18. Dhurat R, Sukesh M, Avhad G, et al. A randomized evaluator blinded study of effect of microneedling in androgenetic alopecia: a pilot study. Int J Trichol. 2013;5(1):6-11.

19. Clementoni MT, Roscher MB, Munavalli GS. Photodynamic photorejuvenation of the face with a combination of microneedling, red light, and broadband pulsed light. Lasers Surg Med. 2010;42:150-159.

20. Torezan L, Chaves Y, Niwa A, et al. A pilot split-face study comparing conventional methyl aminolevulinate-photodynamic therapy (PDT) with microneedling-assisted PDT on actinically damaged skin. Dermatol Surg. 2013;39(8):1197-1201.

21. Badran MM, Kuntsche J, Fahr A. Skin penetration enhancement by a microneedle device (Dermaroller ${ }^{\circledR}$ ) in vitro: dependency on needle size and applied formulation. Eur J Pharm Sci. 2009;36:511-523.

22. Wu Y, Qiu Y, Zhang S, Qin G, Gao Y. Microneedle-based drug delivery: studies on delivery parameters and biocompatibility. Biomed Microdevices. 2008;10:601-610.

23. Gomaa YA, Morrow DI, Garland MJ, et al. Effects of microneedle length, density, insertion time and multiple applications on human skin barrier function: assessments by transepidermal water loss. Toxicol In Vitro. 2010;24:1971-1978.

24. Sand M, Bechara FG, Sand D, Altmeyer P, Hoffmann K. A randomized, controlled, double-blind study evaluating melanin-encapsulated liposomes as a chromophore for laser hair removal of blond, white, and gray hair. Ann Plast Surg. 2007;58:551-554.

25. Leeuw de Jaap MD, Beek Nick van der MD, Neugebauer D. Permanent hair removal of white, grey and light blond hair after laser treatment combined with melanin encapsulated liposomes $\left(\right.$ Lipoxome $\left.^{\circledR}\right)$. Multicare Medical Services, Hilversum, Nederlands.
Clinical, Cosmetic and Investigational Dermatology

\section{Publish your work in this journal}

Clinical, Cosmetic and Investigational Dermatology is an international, peer-reviewed, open access, online journal that focuses on the latest clinical and experimental research in all aspects of skin disease and cosmetic interventions. All areas of dermatology will be covered; contributions will be welcomed from all clinicians and

\section{Dovepress}

basic science researchers globally. This journal is indexed on CAS The manuscript management system is completely online and includes a very quick and fair peer-review system, which is all easy to use. Visit http://www.dovepress.com/testimonials.php to read real quotes from published authors. 